\title{
Disentangling early versus late audiovisual integration in adult ADHD: a combined behavioural and resting-state connectivity study
}

\author{
Marcel Schulze, MSc; Behrem Aslan, MD, PhD; Tony Stöcker, PhD; \\ Rüdiger Stirnberg, PhD; Silke Lux, PhD*; Alexandra Philipsen, MD, PhD*
}

\begin{abstract}
Background: Studies investigating sensory processing in attention-deficit/hyperactivity disorder (ADHD) have shown altered visual and auditory processing. However, evidence is lacking for audiovisual interplay - namely, multisensory integration. As well, neuronal dysregulation at rest (e.g., aberrant within- or between-network functional connectivity) may account for difficulties with integration across the senses in ADHD. We investigated whether sensory processing was altered at the multimodal level in adult ADHD and included resting-state functional connectivity to illustrate a possible overlap between deficient network connectivity and the ability to integrate stimuli. Methods: We tested 25 patients with ADHD and 24 healthy controls using 2 illusionary paradigms: the sound-induced flash illusion and the McGurk illusion. We applied the Mann-Whitney $U$ test to assess statistical differences between groups. We acquired resting-state functional MRIs on a 3.0 T Siemens magnetic resonance scanner, using a highly accelerated 3-dimensional echo planar imaging sequence. Results: For the sound-induced flash illusion, susceptibility and reaction time were not different between the 2 groups. For the McGurk illusion, susceptibility was significantly lower for patients with ADHD, and reaction times were significantly longer. At a neuronal level, resting-state functional connectivity in the ADHD group was more highly regulated in polymodal regions that play a role in binding unimodal sensory inputs from different modalities and enabling sensory-to-cognition integration. Limitations: We did not explicitly screen for autism spectrum disorder, which has high rates of comorbidity with ADHD and also involves impairments in multisensory integration. Although the patients were carefully screened by our outpatient department, we could not rule out the possibility of autism spectrum disorder in some participants. Conclusion: Unimodal hypersensitivity seems to have no influence on the integration of basal stimuli, but it might have negative consequences for the multisensory integration of complex stimuli. This finding was supported by observations of higher resting-state functional connectivity between unimodal sensory areas and polymodal multisensory integration convergence zones for complex stimuli.
\end{abstract}

\section{Introduction}

Our daily-life interaction with our environment is driven by numerous inputs to our sensory systems (e.g., listening to a person speaking while seeing their lip movements and sensing certain odours, all at the same time). To form a unified percept, our brain combines individual streams of stimuli. That process, called multisensory integration, enables us to detect, locate and differentiate external objects more beneficially and precisely than a single stream of input. ${ }^{1}$ Attention depends on stimulus complexity and triggers early or late multisensory integration. When a person is confronted with simple stimuli, bottom-up attentional processing enables early processing; more complex stimuli are processed top-down, because incoming stimuli need to be compared with existing background knowledge via feedback loops to sensory cortices. ${ }^{2-7}$

The process of bottom-up and top-down attentional multisensory integration processing is modulated by adjusting the gain and stimulus saliency between sensory modalities. ${ }^{8}$ In primary sensory areas and superior colliculi, signals are automatically integrated, but top-down attention is necessary in higher-order association areas to properly integrate semantic information, for example. ${ }^{9}$

Attention-deficit/hyperactivity disorder (ADHD) is a neurodevelopmental disorder that is associated with inappropriate levels of inattention, hyperactivity and impulsivity. ${ }^{10}$ Although ADHD has long been considered a childhood

Correspondence to: M. Schulze, Department of Psychiatry and Psychotherapy, University of Bonn, Germany, D-53127 Bonn; marcel.schulze@ukbonn.de

*These authors contributed equally to this work.

Submitted Jan. 20, 2021; Revised May 27, 2021; Accepted Jun. 21, 2021

Cite as: J Psychiatry Neurosci 2021 September 21;46(5). doi: 10.1503/jpn.210017 
disorder, recent studies have shown that $40 \%-50 \%$ of people with ADHD have symptoms in adulthood. ${ }^{11}$ The main symptoms of ADHD and their neuronal underpinnings have been the subject of many investigations, but a neglected area has been multisensory integration, especially in adults.

Children with ADHD show sensory processing deficits and modulatory difficulties via abnormalities in the visual domain; the auditory domain (with increasing issues over time); and with touch, taste, smell, and emotional and social responses. ${ }^{12-14}$ Numerous studies have shown sensory hypersensitivity in children with ADHD, marked primarily by higher distractibility from external stimuli (as a failure to inhibit irrelevant stimuli), especially in the auditory domain. ${ }^{14}$ In adults with ADHD, studies of sensory processing are scarce, but deficits seem to extend into adulthood..$^{15}$ Because unisensory processing is impaired, it raises the question of whether multisensory processing is also affected in adult patients with ADHD.

To the best of our knowledge, only 2 studies have investigated multisensory processing in adults with ADHD. Michalek and colleagues ${ }^{16}$ showed that in adults with ADHD, their understanding of speech in a noisy environment did not improve when speakers' faces were made visible. In other words, although the control group improved their understanding of speech-in-noise, patients with ADHD could not make use of the additional visual information from mouth movements. As mentioned earlier, this was a type of multisensory integration (the integration of auditory speech and visual lip movements is taking place at a higher level of processing, because speech is a complex stimulus). In the other study ${ }^{17}$ — in which patients and controls reacted to parallel presentations of the auditory word "red" and a visual red circle - results showed no difference between groups, suggesting that multisensory integration is not impaired in ADHD. However, as the authors stated, this effect could have been a recognition effect rather than multisensory integration, because the task was solvable without complex cognitive processing. Therefore, further studies are needed to investigate whether multisensory integration is impaired in adult ADHD.

Previous studies have investigated multisensory integration by comparing unisensory to multisensory reaction times, aiming for a behavioural benefit for multisensory integration stimuli (i.e., faster reaction times when successfully integrated). Another way of studying multisensory integration is to create conflicting information across different sensory modalities, leading to perceptual biases or illusions. ${ }^{8}$ This has been done with the sound-induced flash illusion (SIFI) and the McGurk illusion, 2 of the most-used illusion paradigms in multisensory integration research. In the SIFI, visual perception can be influenced by the auditory modality, because the presentation of multiple auditory stimuli can lead to the illusion of several visual stimuli (although the number of visual stimuli actually presented is lower than that of the auditory stimuli). ${ }^{18-20}$ The McGurk illusion is a proxy measure for audiovisual speech integration..$^{21,22}$ By presenting a visual phoneme that is different from an auditory phoneme, the illusion occurs when a completely different phoneme is per- ceived (other than the ones presented) - namely the fused response. The 2 illusions, SIFI and McGurk, differ in stimulus quality, because the SIFI makes use of relatively simple stimuli (e.g., single visual flashes and auditory beep tones) and the McGurk illusion comprises semantic phonemes. However, in both systems the occurrence of the illusion (i.e., the fused response) indicates successful multisensory integration. As mentioned earlier, the complexity of the stimuli influences whether bottom-up (for simpler stimuli) or topdown (for more complex stimuli) processing of multisensory integration is taking place. Applying SIFI and McGurk within a single study allowed us to disentangle early and late multisensory integration. The aim of the current study was to investigate multisensory integration using simple and complex stimuli in the same sample of adults with ADHD. Because the SIFI is driven mainly by auditory processing and ADHD is associated with higher gains and distractibility, particularly in the auditory domain at the unisensory level, we assumed that adults with ADHD and healthy controls would integrate the SIFI illusion in a similar way. In contrast, because of the perceptual hypersensitivity outlined above, we hypothesized that adults with ADHD would show deficient multisensory integration for more complex stimuli in the McGurk illusion.

ADHD is characterized by structural and functional impairments in brain regions associated with multisensory integration. The temporoparietal junction, insula, anterior cingulum, inferior frontal gyrus, mediofrontal gyrus and middle temporal gyrus are all associated with polysensory responses and may be the loci of multisensory integration. ${ }^{23}$ These regions are also part of the default mode network, the dorsal attention network and the salience networks, ${ }^{24,25}$ which have been investigated frequently in resting-state functional MRI studies. In ADHD, most of the regions are dysregulated at rest, showing aberrant within- or between-network functional connectivity, which may also imply difficulties with multisensory integration. Therefore, we included restingstate functional connectivity in the current study to illustrate a possible overlap between network connectivity and the ability to integrate stimuli. We assumed altered resting-state functional connectivity at polysensory brain areas.

\section{Methods}

\section{Participants}

We recruited 25 patients with ADHD (6 female; age [mean \pm standard deviation] $30.08 \pm 9.3$ years $)$ at our psychiatric outpatient department. We recruited 24 healthy controls (9 female; age $26.88 \pm 6.3$ years) using bulletin boards. All participants provided written consent to take part, and the study was approved by the ethics committee of the medical faculty of the university of Bonn (166/18). Patients with ADHD who were taking stimulant medication were asked to discontinue the medication at least 24 hours before the experiment.

All patients had received a diagnosis of ADHD according to international guidelines, ${ }^{26,27}$ based on DSM-5 diagnostic 
criteria. Patients were diagnosed by a specialist psychiatrist after a detailed clinical and psychosocial interview that integrated somatic differential diagnosis, patients' psychiatric and developmental history, and observer reports.

To further assess ADHD symptoms, we used the Conners Adult ADHD Rating Scales (CAARS), long version, selfrated. ${ }^{28}$ We used the Wender Utah Rating Scale (WURS-k) to rate ADHD symptoms in childhood. ${ }^{29}$ We assessed patients for comorbid psychiatric disorders using the Structured Clinical Interview for DSM-5, short interview. ${ }^{30}$

Participants provided a self-rating of depressive symptoms using the Beck Depression Inventory. ${ }^{31}$ We used the Edinburgh Handedness Questionnaire ${ }^{32}$ to confirm that the right hand was the most dominant for each participant. This was important because responses during the experiment were made with the right hand on a controller.

\section{Behavioural experiments}

Participants were seated approximately $60 \mathrm{~cm}$ in front of a screen in a light-dimmed room. Auditory stimuli were presented via headphones with the sound pressure level adjusted for each participant.

\section{Sound-induced flash illusion}

In the SIFI, brief auditory stimuli influence the number of perceived visual stimuli. The illusion occurs as a result of an incongruent number of auditory beeps (e.g., 2 beeps) and visual flashes (e.g., 1 flash), leading to the impression that 2 visual flashes have been presented. Beeps consisted of $1 \mathrm{kHz}$ tones presented for $10 \mathrm{~ms}$ (1 ms ramp), and flashes consisted of white circles (sized $0.75^{\circ}$ ) presented on a black background for $16 \mathrm{~ms}$. The auditory stimulus was created using MATLAB release 2018b (MathWorks), and flashes were created using Presentation (version 22.1 Neurobehavioural Systems). In the experiment, 2 unimodal and 3 bimodal conditions were presented. The unimodal conditions consisted of 1 flash (1F) and 2 flashes (2F), respectively. Bimodal conditions consisted of 1 flash (1F) with 1 beep (1B) and 2 flashes (2F) with 2 beeps (2B). The SIFI condition consisted of an incongruent number of stimuli: 1 flash (1F) and 2 beeps (2B). Whenever 2 stimuli of the same category (e.g., 2 beeps) were presented, stimulus onset asynchronies were chosen between $70 \mathrm{~ms}$ and $420 \mathrm{~ms}$ (increases in steps of $50 \mathrm{~ms}$ ). Each condition involved 160 trials (20 trials per stimulus onset asynchrony), and each trial began with a fixation cross for $1000 \mathrm{~ms}$. Conditions were presented randomly with a break after half of the trials had been completed. Participants were instructed to report the number of perceived flashes as quickly as possible using a controller.

\section{McGurk illusion}

To measure audiovisual speech intelligibility, we took existing stimuli from the Oldenburg Audio Visual Speech Stimuli data set. ${ }^{33}$ In this scenario, 6 speakers ( 3 females) were chosen to present syllables (e.g., "ba," "ga") in 4 different conditions (120 trials each): unimodal auditory, unimodal visual, bimodal congruent and bimodal incongruent. Participants were instructed to report what they heard in bimodal condi- tions and to report the corresponding sense for unimodal conditions (lip reading for the visual condition and hearing for the auditory condition). After each clip, a 4-alternative forced-choice task was presented, consisting of auditory, visual, and 2 possible fused response options. Conditions were presented randomly across 6 blocks.

\section{Statistical analysis}

We compared all demographic variables between groups using independent $t$ tests.

We calculated susceptibility scores individually for the SIFI and the McGurk illusion, as well as the number of correct responses in control conditions. We defined correct responses in the SIFI as the correct number of flashes seen, and in the McGurk illusion as the heard syllable (except for the unimodal visual condition, in which lip reading was taking place). Outliers on reaction time data were detected using the MATLAB build-in function "rmoutliers." An outlier was defined as a finding with a value above 3 median absolute deviations.

We used nonparametric Mann-Whitney $U$ tests to compare response scores (including susceptibility scores and number of correct responses) and reaction times between groups. We used Pearson correlation for CAARS inattention/ susceptibility scores. We used childhood ADHD symptoms (WURS-k score) as a regressor for susceptibility to the McGurk illusion.

\section{Neuroimaging protocol}

We acquired structural images on a 3.0 T MRI scanner (Magnetom Skyra, Siemens Healthineers) using a 32-channel head coil. We acquired magnetization-prepared rapid gradient echo (MP-RAGE) $T_{1}$-weighted images with an acquisition time of 2 min $40 \mathrm{~s}$ using controlled aliasing in parallel imaging results in higher acceleration (CAIPIRINHA) and elliptical sampling (repetition time $2500 \mathrm{~ms}$, echo time $3.55 \mathrm{~ms}$, inversion time $1100 \mathrm{~ms}$, flip angle $7^{\circ}$, matrix size $256 \times 256 \times$ 176 , voxel size $1.0 \times 1.0 \times 1.0 \mathrm{~mm}^{3}$, sagittal slice orientation, slice parallel imaging acceleration factor 3 , CAIPI shift 1 , Turbofactor 192). ${ }^{34,35}$ We acquired functional resting-state images consisting of 850 volumes with a rapid 3-dimensional echo planar imaging sequence that combined CAIPIRINHA, semielliptical sampling and water-selective fat suppression (repetition time $760 \mathrm{~ms}$, echo time $30 \mathrm{~ms}$, flip angle $18^{\circ}$, matrix size $84 \times 84 \times 56$, voxel size $2.5 \times 2.5 \times 2.5 \mathrm{~mm}^{3}$, oblique-axial slice orientation approximately along the anterior-posterior commissure, anterior-posterior phase encoding direction, slice parallel imaging acceleration factor 4 , CAIPI shift 2). ${ }^{36}$

\section{Image processing}

Data were preprocessed and analyzed using MATLAB release 2018b and SPM12 (www.fil.ion.ucl.ac.uk/spm/ software/spm12/). Functional images were realigned using a 6-parameter rigid body transformation. We performed motion correction and susceptibility distortion correction using a voxel displacement map. Further preprocessing included 
coregistration to a structural scan, spatial normalization to Montreal Neurological Institute space and spatial smoothing (Gaussian kernel, $8 \mathrm{~mm}$ full width at half maximum).

\section{Resting-state functional connectivity}

We analyzed seed-to-voxel functional connectivity using the CONN 19.c toolbox (https:/ / web.conn-toolbox.org/home).

Using the default processing parameters in CONN, we regressed out potential confounding effects (white matter, cerebrospinal fluid and motion) from the functional data using an anatomic component-based noise correction procedure (aCompCor). We also used a band-pass filter $(0.008-0.09 \mathrm{~Hz})$ to further reduce noise such as respiration, pulsation and scanner drift. To detect motion outliers, we performed linear detrending and frame censoring using image-scrubbing Artifact Detection Tools. We generated individual correlation maps by extracting the mean blood-oxygen-level-dependent (BOLD) time course from each region of interest and calculating correlation coefficients using the BOLD time course for each voxel throughout the whole brain. We chose regions of interest based on the literature, including the default mode network (e.g., inferior parietal cortex, anterior cingulate cortex, hippocampus, orbitofrontal cortex), ${ }^{37}$ the salience network (e.g., insula) ${ }^{38}$ and the cognitive control networks (e.g., precentral gyrus, supramarginal gyrus, calcarine sulcus). ${ }^{39}$ We then used these maps for second-level analyses with a 2-sided independent $t$ test to investigate differences in seedto-voxel connectivity between patients with ADHD and controls. We also performed an analysis of covariance, controlling for McGurk susceptibility. Statistical significance was set at voxel-wise $p<0.001$ (uncorrected), before false discovery rate correction was applied at the cluster level $(p<0.05)$.

\section{Results}

\section{Behavioural data}

For a demographic overview, see Table 1. We found no differences between groups in terms of age or sex.

\section{Sound-induced flash illusion}

Susceptibility to the SIFI $(1 \mathrm{~F} / 2 \mathrm{~B})$ revealed no differences between groups (median: ADHD 6.9\%, control 10.1\%; $U=$ 237.0, $p=0.41, r=-0.12)$. We found no differences in performance for the control conditions or for reaction times between groups (median: ADHD $777 \mathrm{~ms}$, control $693 \mathrm{~ms} ; U=$ 222.0, $p=0.25, r=-0.17$ ) in cases of successful multisensory integration. We also found no reaction differences in control conditions (Figure 1).

\section{McGurk illusion}

The Mann-Whitney $U$ test indicated significantly more fused responses for control participants in the bimodal incongruent condition (median: ADHD 5.83\%, control 44.2\%; $U=160.5$, $p=0.022, r=-0.34$ ). With respect to the instructions to report the heard phenomenon, patients with ADHD relied correctly on the auditory response in the 4-alternative forced-choice task (median: ADHD 93.3\%, control 51.7\%; $U=166.0, p=0.03$,

\begin{tabular}{|c|c|c|}
\hline Characteristic $^{*}$ & ADHD† & Controls \\
\hline Patients (female), $n$ & $25(6)$ & $24(9)$ \\
\hline Age, yr & $30.08 \pm 9.3$ & $26.88 \pm 6.3$ \\
\hline CAARS score, inattention & $19.8 \pm 6.5 \ddagger$ & $6.29 \pm 4.8$ \\
\hline CAARS score, hyperactivity/impulsivity & $20.32 \pm 7.9 \ddagger$ & $7.58 \pm 5.8$ \\
\hline WURS-k score & $39.8 \pm 11.8 \ddagger$ & $11.42 \pm 7.2$ \\
\hline BDI score & $9.44 \pm 8.2 \ddagger$ & $2.04 \pm 1.9$ \\
\hline Previous substance abuse, $n$ & 9 & - \\
\hline \multicolumn{3}{|l|}{ Lifetime comorbidities, $n$} \\
\hline Major depression & 5 & - \\
\hline Generalized anxiety disorder & $<5$ & - \\
\hline PTSD & $<5$ & - \\
\hline Borderline personality disorder & $<5$ & - \\
\hline \multicolumn{3}{|l|}{ Medication, $n$} \\
\hline Methylphenidate & 5 & - \\
\hline Elontril & $<5$ & - \\
\hline Sertraline & $<5$ & - \\
\hline Triptane & $<5$ & - \\
\hline Gestagen & $<5$ & - \\
\hline L-thyroxine & $<5$ & - \\
\hline \multicolumn{3}{|c|}{$\begin{array}{l}\text { ADHD = attention-deficit/hyperactivity disorder; BDI = Beck Depression Inventory; } \\
\text { CAARS = Conners' Adult ADHD Rating Scale; PTSD = posttraumatic stress disorder; } \\
\text { WURS-k = Wender-Utah-Rating-Scale. } \\
\text { *Values are mean } \pm \text { standard deviation or } n \text {. } \\
\text { †Values of less than } 5 \text { have been rounded to protect participant privacy. } \\
\ddagger \text { Significantly different from controls at } p=0.01 \text {. }\end{array}$} \\
\hline
\end{tabular}

$r=-0.32$; Figure 1). We found no differences in the bimodal congruent and unimodal auditory/visual control conditions. If multisensory integration took place in the bimodal incongruent condition, reaction times differed significantly between groups (median: ADHD $1260 \mathrm{~ms}$, control $582 \mathrm{~ms}$; $U=$ 41.0, $p<0.01, r=-0.56)$. Compared to controls, patients with ADHD had slower reaction times across all control conditions, although this finding was not significant.

\section{ADHD characteristics: McGurk fused scores}

The occurrence of multisensory integration was inversely related to inattention $\left(r_{45}=-0.49, p=0.001\right.$; Figure 2$)$ and to hyperactivity/impulsivity $\left(r_{45}=-0.37, p=0.12\right)$. Furthermore, we calculated a linear regression to predict fused McGurk scores based on the severity of childhood ADHD (WURS-k). We found that childhood ADHD severity could negatively predict multisensory integration performance on the McGurk illusion $\left(R^{2}=0.139 ; F_{1,44}=7.09, p<0.001\right.$; Figure 3$)$.

\section{Resting-state functional connectivity}

\section{Patients with ADHD versus controls}

Seed-to-voxel correlation analysis revealed that patients with ADHD elicited higher brain functional connectivity between the left insula and the left lingual gyrus, the right planum polare and the primary motor cortex, the right inferior colliculus and the left precuneus, and the left hippocampus and the left parietal inferior gyrus (Figure 4). We also found higher connectivity between the left Heschl gyrus and the visual cortex, and the inferior temporal gyrus and the pars triangularis. 
A Sound-induced flash illusion

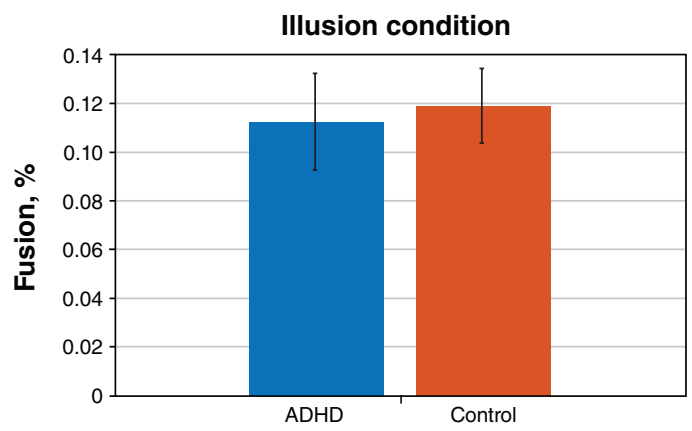

Control condition

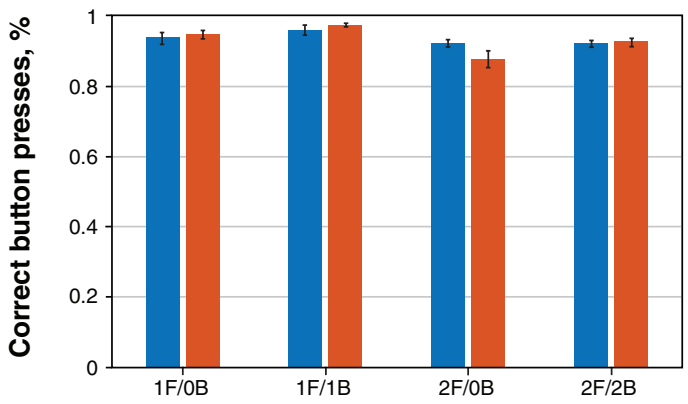

B McGurk illusion

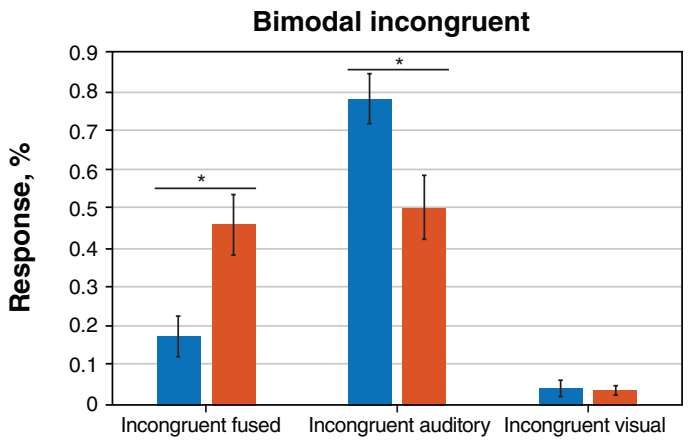

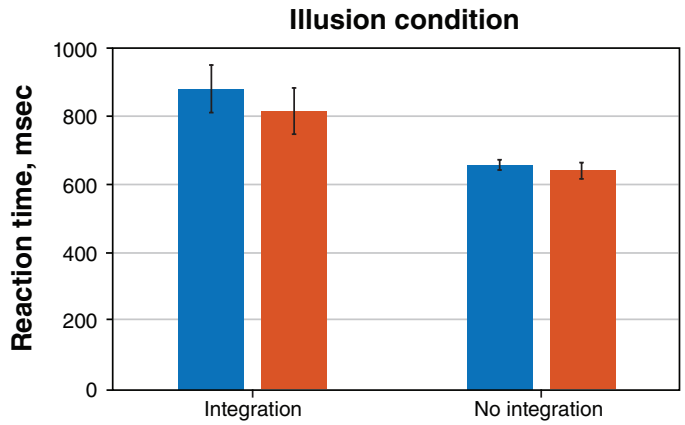

Control condition
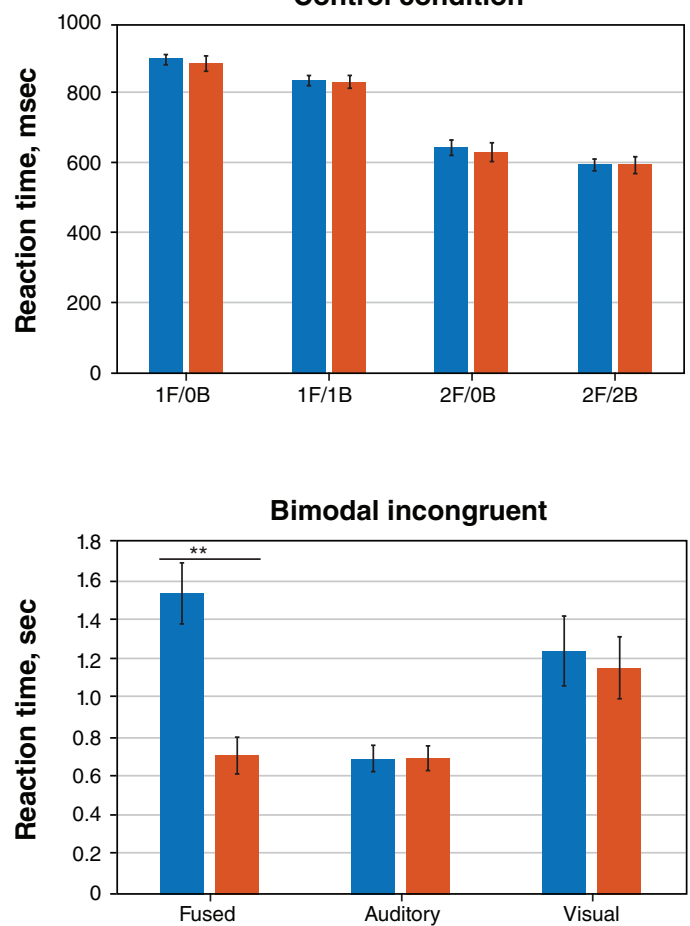

Figure 1: (A) Sound-induced flash illusion. Upper left, fusion rates for the bimodal incongruent condition (1 flash/2 beeps); upper right, reaction times compared for the bimodal incongruent condition ( $1 \mathrm{flash} / 2$ beeps) for integration and no integration; lower left, percentage of correct button presses for control conditions; lower right, reaction times for control conditions. (B) McGurk illusion. Left, comparison of responses to the bimodal incongruent condition: fused response (successful integration), auditory input (heard tone) or visual input (lip movements). Right, comparison of reaction times for successful integration (fused) or auditory or visual answers. ${ }^{*} p=0.05 ;{ }^{* *} p=0.01$. B $=$ beep; $\mathrm{F}=$ flash.

\section{McGurk and resting-state functional connectivity}

Correlational analysis across the entire sample revealed a negative relationship between McGurk illusion scores and resting-state functional connectivity for the inferior frontal gyrus, the anterior cingulum and the precuneus (Figure 4). Resting-state functional connectivity was negatively correlated with the McGurk illusion score for the parahippocampal cortex and the frontal orbital cortex. Selecting the hippocampus as a seed region revealed negative associations for the inferior frontal gyrus, the right insula and the fusiform gyrus.
In the middle temporal gyrus, we found negative associations for the primary auditory cortex, the inferior frontal gyrus, the precentral gyrus and the fusiform gyrus.

\section{Discussion}

This was the first study to comprehensively investigate multisensory integration with simple (SIFI) and complex (McGurk illusion) stimuli separately in the same sample of adults with ADHD compared to a healthy control group. To 


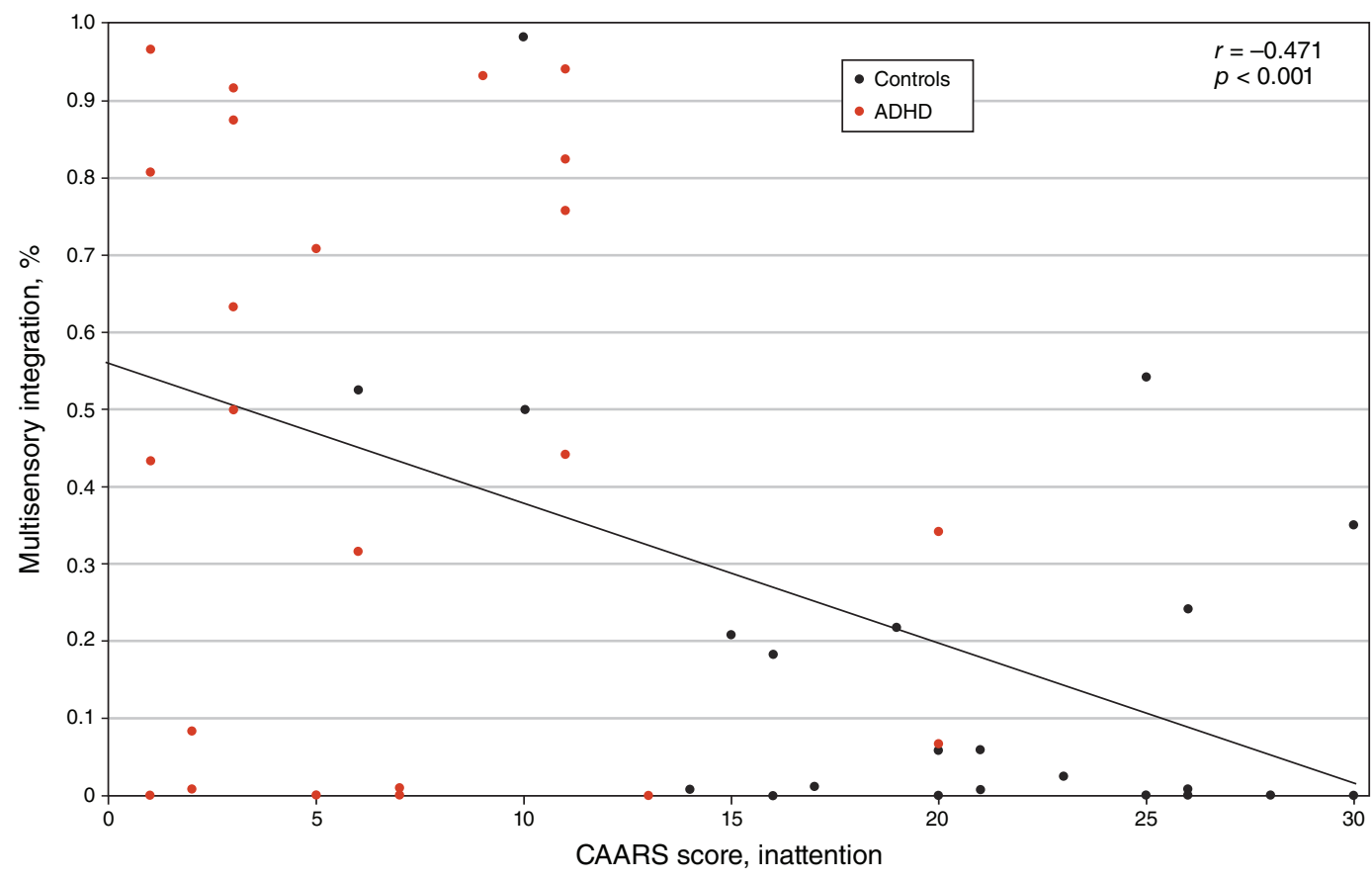

Figure 2: Correlation between CAARS inattention scores and fused (multisensory integration) responses to the McGurk illusion. ADHD = attention-deficit/hyperactivity disorder; CAARS = Conners' Adult ADHD Rating Scale.

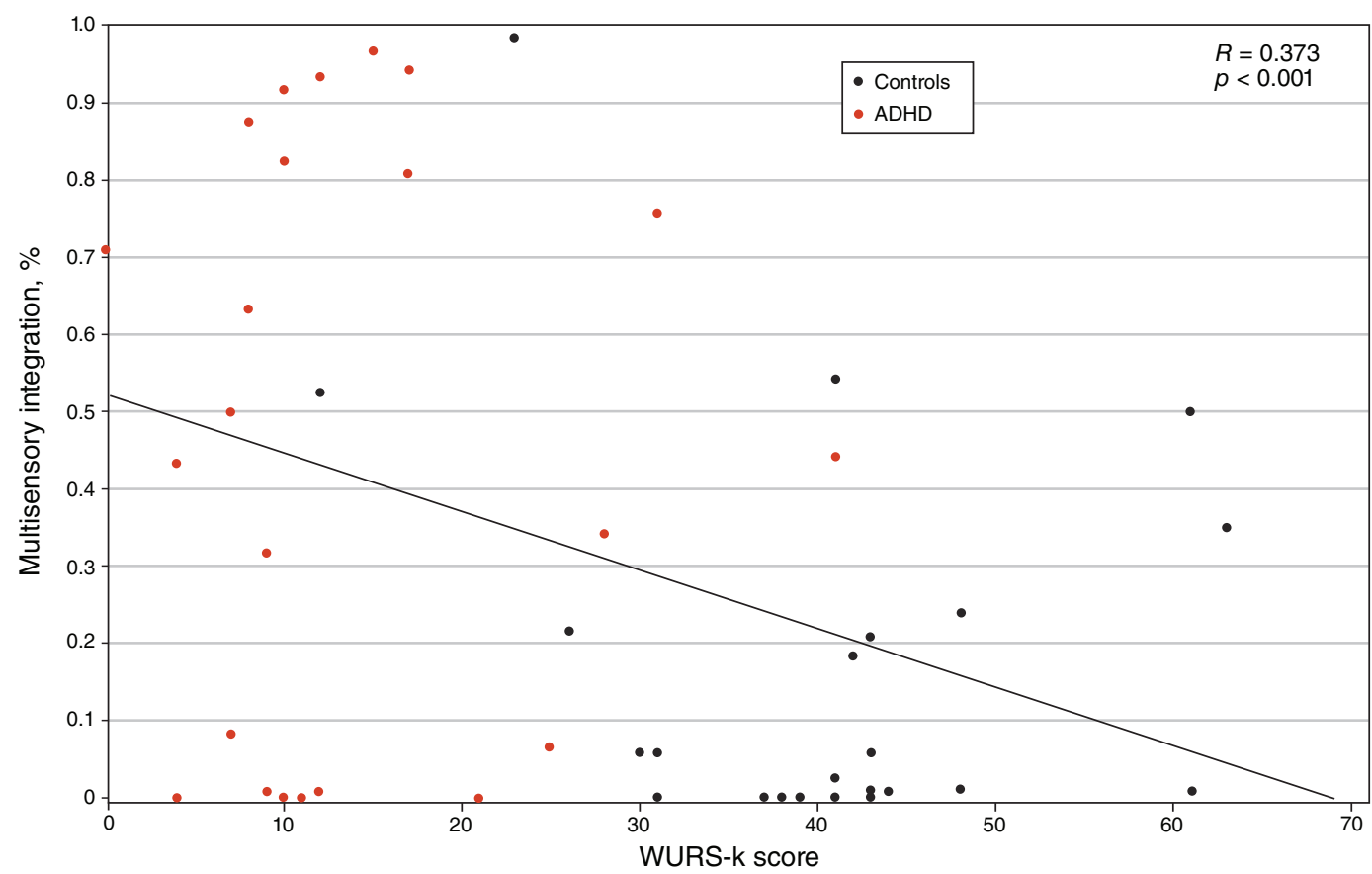

Figure 3: Linear regression with severity of childhood ADHD (WURS-k score) as a predictor of fused (multisensory integration) responses to the McGurk illusion. ADHD = attention-deficit/hyperactivity disorder; WURS-k = Wender Utah Rating Scale. 


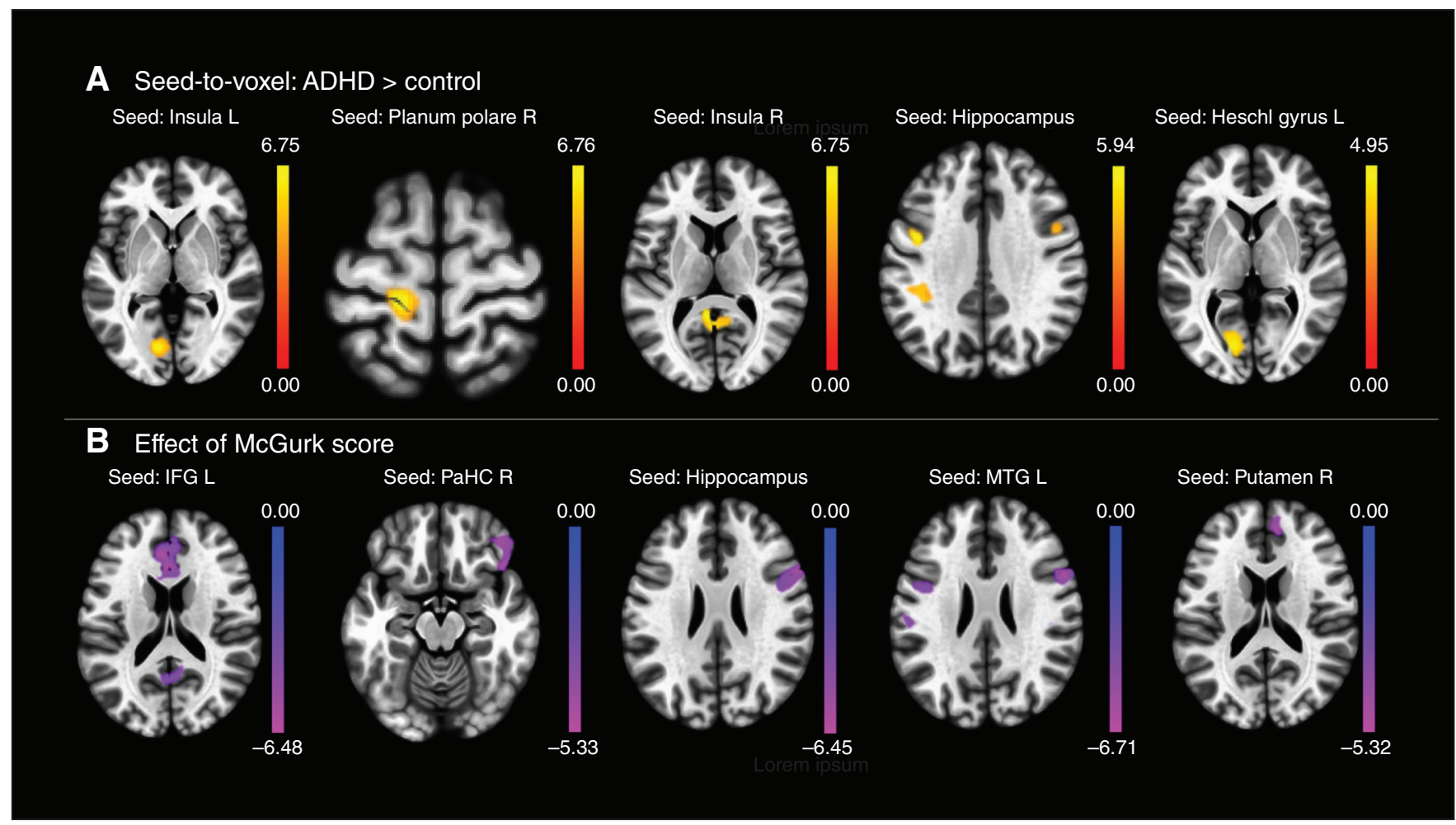

Figure 4: Resting-state functional connectivity. (A) Enhanced resting-state functional connectivity in patients with ADHD compared to healthy controls. (B) Correlation between McGurk scores and resting-state functional connectivity across the entire study sample. False discovery rate correction was applied at the cluster level $(p<0.05)$. ADHD = attention-deficit/hyperactivity disorder; IFG = inferior frontal gyrus; $\mathrm{MTG}=$ middle temporal gyrus; $\mathrm{PaHC}=$ parahippocampal gyrus.

identify possible overlaps between pathophysiologic restingstate activity and multisensory integration, we calculated seed-to-voxel resting-state functional connectivity. Our assumption was that adult patients with ADHD would successfully integrate simple stimuli but fail at multisensory integration for complex stimuli. Not surprisingly, we found no difference in terms of fused responses and reaction times with the SIFI. In the McGurk illusion, patients with ADHD experienced significantly fewer illusions because of a sensory preference for auditory stimuli in multimodal scenarios. In trials in which patients with ADHD successfully integrated stimuli, they were significantly slower than controls. Furthermore, childhood severity of ADHD predicted multisensory integration on the McGurk illusion and was inversely associated with inattention. At a neuronal level, resting-state functional connectivity in patients with ADHD was more highly regulated in polymodal regions known to play a role in binding unimodal sensory inputs from different modalities to enable sensory-to-cognition integration.

Although patients with ADHD were able to integrate signals in the SIFI at a similar level to controls, overall integration rates across all participants were relatively low. The optimal time interval for the occurrence of the illusion is $100 \mathrm{~ms}$ before or after the onset of the flash. ${ }^{40}$ The overall low fused response rates we found in the present study might have been caused by asynchronies that were too wide (maximum asynchrony of $420 \mathrm{~ms}$ ), although several studies have shown integration effects for asynchronies up to $600 \mathrm{~ms}^{40-42}$ Sensory processing in ADHD is marked by hypersensitivity, especially for the auditory sense. ${ }^{43,44}$ Although the SIFI is an illusion in which visual processing is influenced by auditory processing, it is possible that auditory hypersensitivity is beneficial for integration in that scenario. However, possible enhanced bottom-up sensory processing could have consequences for later processing stages (e.g., improper filtering of incoming stimuli may have consequences for attentional allocation at later stages). Our findings for the SIFI were in line with those of McCracken and colleagues, ${ }^{17}$ who found that patients with ADHD showed faster response times in multisensory integration conditions compared to unisensory response latencies. However, they also found the longest response latencies for the auditory-only condition (a verbalization of the word "red"). This finding could indicate that more complex stimuli, which need higher-order processing, are disadvantageously regulated in terms of attentional allocation. By disentangling multisensory integration for simple and complex stimuli, we were able to demonstrate that patients with ADHD had difficulty integrating speech-like phonemes. When these patients did not integrate the incongruent stimuli to a new, single percept, they relied more on the auditory modality (i.e., they reported significantly more auditory phonemes) than the healthy controls. All participants were instructed to report the auditory phoneme primarily in the multisensory integration condition, which 
could mean that patients with ADHD simply followed the instructions more strictly than the controls. Alternatively, it also raises the possibility that auditory hypersensitivity is the primary sense ADHD patients rely on in multisensory integration scenarios. In other words, bottom-up sensitivity, which might be beneficial for simple stimuli, could hinder successful integration for complex stimuli, because topdown allocation does not take place properly. This assumption is also in line with the response latencies we found in the McGurk illusion. When patients with ADHD integrated successfully, response latencies were significantly longer than those of controls. Furthermore, we know that auditory information directly influences and enhances visual processing in a bottom-up fashion. ${ }^{45}$ This process boosts multisensory integration in terms of attentional capturing, whereas a single sensory event would not capture attention. ${ }^{46}$ In our study, an interpretation is that in ADHD patients this boosting process was deficient for complex stimuli, because attention was captured at later latencies or not at all compared to healthy controls. This point of view would be supported by the findings of Michalek and colleagues, ${ }^{16}$ who investigated speech-in-noise understanding for a potential benefit when visual information was added. In patients with ADHD, the inclusion of visual information did not facilitate higher speech-in-noise understanding to the same extent as for controls.

The exact factors that lead to multisensory integration for complex stimuli in some trials and not in others remain elusive. One point of view is that selective attention - necessary for multisensory integration - is modulated by the prestimulus rate of cortical networks. Dependent on the oscillatory state (either synchronized across networks or not), perceptual binding in multisensory integration scenarios could occur. ${ }^{47-49}$ Attention can act as a modulator for oscillatory activity and is itself determined by trial-by-trial variability. ${ }^{49}$ Larger trial-by-trial neural response variability has been reported for visual and auditory unisensory events in ADHD. ${ }^{50}$ Although the effect of such enhanced neural variability on multisensory integration in ADHD has not yet been investigated, we can assume that enhanced trial-by-trial variability could also play a role in deficient multisensory integration. Such higher neuronal fluctuations are associated with deficiency in the default mode network - an intrinsic, spontaneous activation of brain areas at rest, usually suppressed in the presence of a task. ${ }^{51}$ In ADHD, disrupted activity in the default mode network has been found (i.e., increased withinnetwork connectivity at the temporal pole-inferior frontal gyrus and decreased within-network connectivity to the posterior cingulate cortex). ${ }^{52}$ In the present study, we found higher resting-state functional connectivity in patients with ADHD, not only in areas usually associated with the functioning of the default mode network, but also in areas related to sensory processing and sensory integration. Heschl's gyrus showed higher connectivity to the visual cortex, which could indicate abnormal regulation of the influence on sensory processing of the auditory cortex to the visual cortex as described above. In general, multisensory integration is a complex interplay of regions throughout the brain. The main loci of multisensory integration are the temporoparietal junction, the middle temporal gyrus, the middle frontal gyrus, the inferior frontal gyrus, the anterior cingulate cortex, the insula and the hippocampus, all of which receive inputs from sensory modalities. ${ }^{23}$ Our analysis revealed higher resting-state functional connectivity between sensory areas and frontal areas. Because of higher network activity at rest, this result could mirror disrupted communication from areas of lower sensory integration to areas of higher sensory integration (e.g., the insula as a causal control hub in multisensory attention). ${ }^{53}$ Furthermore, the planum temporale, associated with complex auditory processing, also showed hyperconnectivity to the insula ${ }^{54,55}$ in our sample of adults with ADHD. We can only speculate whether this result reflects higher gains and distractibility in the auditory sense at the network level for patients with ADHD. The middle temporal gyrus is frequently reported as a multimodal area that reflects the integration of sensory-specific input for complex objects. ${ }^{56}$ Taking the fused scores of the McGurk illusion as a regressor, the seeded middle temporal gyrus was inversely associated with resting-state functional connectivity to the primary auditory cortex, the inferior frontal gyrus, the precentral gyrus and the fusiform gyrus. This result further supports the assumption that communication from lower sensory areas to sensoryintegrating areas might be disrupted in ADHD. Simultaneously, the left putamen, the left temporal pole, the right caudate and the left inferior frontal gyrus were hypoactivated in patients with ADHD in a task-based functional metaanalysis, further indicating an overlap between ADHD symptomatology, brain activation and multisensory integration. ${ }^{57}$ Overall, our results were in line with findings for autism spectrum disorder, a clinical disorder that is often comorbid with $\mathrm{ADHD}^{58}$ and also involves difficulties in integrating complex signals, ${ }^{59,60}$ although some studies have reported integration rates similar to healthy controls. ${ }^{61,62}$ At a neuronal level, a stepwise functional connectivity analysis revealed hyperconnectivity between the sensory unimodal and default mode networks. ${ }^{63}$ In contrast to our results, hypoconnectivity of sensory unimodal areas to frontoparietal areas has been reported in autism spectrum disorder.

\section{Limitations}

We recruited a relatively small sample size. As well, in our screening assessment we did not account specifically for autism spectrum disorder, which has high comorbidity with ADHD and also involves impairments for multisensory integration. Although patients with ADHD were carefully screened by our outpatient department, we cannot rule out the possibility of autism spectrum disorder in some participants.

\section{Conclusion}

Adult patients with ADHD showed unimpaired bottom-up sensory integration of simple stimuli, but impaired topdown multisensory integration of more complex stimuli. We concluded that sensory impairments at a unisensory level led to deficient multisensory integration for complex 
stimuli, indicating a failure of late attentional deployment. This conclusion was supported by findings of higher resting-state functional connectivity from unimodal sensory areas to polymodal convergence zones for multisensory integration. Future studies are needed to clarify whether deficient multisensory integration for complex stimuli can account for ADHD core symptoms, or whether these are independent constructs.

Affiliations: From the Department of Psychiatry and Psychotherapy, University of Bonn, Bonn, Germany (Schulze, Aslan, Lux, Philipsen); Biopsychology and Cognitive Neuroscience, Faculty of Psychology and Sports Science, Bielefeld University, Bielefeld, Germany (Schulze); the German Centre for Neurodegenerative Diseases (DZNE), Bonn, Germany (Stöcker, Stirnberg); and the Department of Physics and Astronomy, University of Bonn, Bonn, Germany (Stöcker).

Competing interests: A. Philipsen has served on advisory boards, given lectures, performed phase 3 studies, or received travel grants within the last 5 years from Eli Lilly and Co, Lundbeck, MEDICE Arzneimittel, Pütter GmbH and Co KG, Novartis, Servier, Shire/ Takeda and Boehringer; she has authored books and articles on ADHD published by Elsevier, Hogrefe, Schattauer, Kohlhammer, Karger, Oxford Press, Thieme and Springer.

Contributors: S. Lux and A. Philipsen designed the study. B. Aslan, T. Stöcker and R. Stirnberg acquired the data, which M. Schulze analyzed. M. Schulze, R. Stirnberg and A. Philipsen wrote the article, which B. Aslan, T. Stöcker and S. Lux reviewed. All authors approved the final version to be published and can certify that no other individuals not listed as authors have made substantial contributions to the paper.

Content licence: This is an Open Access article distributed in accordance with the terms of the Creative Commons Attribution (CC BY-NC-ND 4.0) licence, which permits use, distribution and reproduction in any medium, provided that the original publication is properly cited, the use is noncommercial (i.e., research or educational use), and no modifications or adaptations are made. See: https:/ / creativecommons.org/licenses/by-nc-nd/4.0/

\section{References}

1. Dhamala M, Assisi CG, Jirsa VK, et al. Multisensory integration for timing engages different brain networks. Neuroimage 2007; 34:764-73.

2. Talsma D. Predictive coding and multisensory integration: an attentional account of the multisensory mind. Front Integr Nuerosci 2015;9:19.

3. van Wassenhove V, Grant KW, Poeppel D. Temporal window of integration in auditory-visual speech perception. Neuropsychologia 2007;45:598-607.

4. Stein BE, Stanford TR. Multisensory integration: current issues from the perspective of the single neuron. Nat Rev Neurosci 2008; 9:255-66.

5. Holmes NP. The principle of inverse effectiveness in multisensory integration: some statistical considerations. Brain Topogr 2009.

6. Noesselt T, Tyll S, Boehler CN, et al. Sound-induced enhancement of low-intensity vision: multisensory influences on human sensoryspecific cortices and thalamic bodies relate to perceptual enhancement of visual detection sensitivity. J Neurosci 2010;30:13609-23.

7. Rach S, Diederich A, Colonius H. On quantifying multisensory interaction effects in reaction time and detection rate. Psychol Res 2011;75:77-94.

8. Choi I, Lee JY, Lee SH. Bottom-up and top-down modulation of multisensory integration. Curr Opin Neurobiol 2018;52:115-122.

9. MacAluso E, Noppeney U, Talsma D, et al. The curious incident of attention in multisensory integration: bottom-up vs. top-down. Multisens Res 2016;29:557-83.

10. Willcutt EG. The prevalence of DSM-IV attention-deficit/hyperactivity disorder: a meta-analytic review. Neurotherapeutics 2012;9:490-9.
11. Sibley MH, Mitchell JT, Becker SP. Method of adult diagnosis influences estimated persistence of childhood ADHD: a systematic review of longitudinal studies. Lancet Psychiatry 2016;3:1157-65.

12. Dunn W, Bennett D. Patterns of sensory processing in children with attention deficit hyperactivity disorder. Occup Ther J Res 2002; 22:4-15.

13. Cheung PPP, Siu AMH. A comparison of patterns of sensory processing in children with and without developmental disabilities. Res Dev Disabil 2009;30:1468-80.

14. Ghanizadeh A. Sensory processing problems in children with ADHD, a systematic review. Psychiatry Investig 2011;8:89-94.

15. Bijlenga D, Tjon-Ka-Jie JYM, Schuijers F, et al. Atypical sensory profiles as core features of adult ADHD, irrespective of autistic symptoms. Eur Psychiatry 2017;43:51-57.

16. Michalek AMP, Watson SM, Ash I, et al. Effects of noise and audiovisual cues on speech processing in adults with and without ADHD. Int J Audiol 2014;53:145-52.

17. McCracken HS, Murphy BA, Glazebrook CM, et al. Audiovisual multisensory integration and evoked potentials in young adults with and without attention-deficit/hyperactivity disorder. Front Hum Neurosci 2019;13.

18. Shams L, Kamitani Y, Shimojo S. Illusions: what you see is what you hear. Nature 2000;408:788.

19. Hirst RJ, McGovern DP, Setti A, et al. What you see is what you hear: twenty years of research using the sound-induced flash illusion. Neurosci Biobehav Rev 2020;118:759-74.

20. Keil J. Double flash illusions: current findings and future directions. Front Neurosci 2020;14:298.

21. McGurk H, Macdonald J. Hearing lips and seeing voices. Nature 1976;264:746-8.

22. Alsius A, Paré M, Munhall KG. Forty years after hearing lips and seeing voices: the McGurk effect revisited. Multisens Res 2018;31:111-44.

23. Tanaka E, Kida T, Kakigi R, et al. Neuroscientific evidence for multisensory convergence and interaction. J Phys Fit Sports Med 2017;6:301-10.

24. Cortese S, Aoki YY, Itahashi T, et al. Systematic review and metaanalysis: resting state functional magnetic resonance imaging studies of attention-deficit/hyperactivity disorder. J Am Acad Child Adolesc Psychiatry 2020;60:61-75.

25. Gao Y, Shuai D, Bu X, et al. Impairments of large-scale functional networks in attention-deficit/hyperactivity disorder: a meta-analysis of resting-state functional connectivity. Psychol Med 2019;49:2475-85.

26. Heinzl S. Neue S3-Leitlinie ADHS bei Kindern, Jugendlichen und Erwachsenen. DNP Der Neurologe \& Psychiater 2018;19:60.

27. National Institute for Health and Care Excellence (NICE). Attention deficit hyperactivity disorder: diagnosis and management (NICE guideline). London: NICE; 2018

28. Christiansen H, Kis B, Hirsch O, et al. German validation of the Conners Adult ADHD Rating Scales (CAARS) II: reliability, validity, diagnostic sensitivity and specificity. Eur Psychiatry 2012;27:321-8.

29. Retz-Junginger P, Retz W, Blocher D, et al. Wender Utah rating scale (WURS-k): die deutsche kurzform zur retrospektiven erfassung des hyperkinetischen syndroms bei erwachsenen. Nervenarzt 2002;73:830-8.

30. First MB, Spitzer RL, Williams JBW, et al. Structured Clinical Interview for DSM-IV (SCID). Washington (DC): American Psychiatric Association; 1995.

31. Beck AT, Steer RA, Brown GK. Manual for the Beck depression inventory-II. San Antonio (TX): Psychological Corporation; 1996.

32. Oldfield RC. The assessment and analysis of handedness: the Edinburgh inventory. Neuropsychologia 1971;9:97-113.

33. Stropahl M, Schellhardt S, Debener S. McGurk stimuli for the investigation of multisensory integration in cochlear implant users: the Oldenburg Audio Visual Speech Stimuli (OLAVS). Psychon Bull Rev 2017;24:863-72.

34. Breuer FA, Blaimer M, Mueller MF, et al. Controlled aliasing in volumetric parallel imaging (2D CAIPIRINHA). Magn Reson Med 2006;55:549-56

35. Brenner D, Stirnberg R, Pracht ED, et al. Two-dimensional accelerated MP-RAGE imaging with flexible linear reordering. MAGMA 2014;27:455-62.

36. Stirnberg R, Huijbers W, Brenner D, et al. Rapid whole-brain resting-state $\mathrm{fMRI}$ at $3 \mathrm{~T}$ : efficiency-optimized three-dimensional EPI versus repetition time-matched simultaneous-multi-slice EPI. Neuroimage 2017;163:81-92. 
37. Raichle ME. The brain's default mode network. Annu Rev Neurosci 2015;38:433-47.

38. Goulden N, Khusnulina A, Davis NJ, et al. The salience network is responsible for switching between the default mode network and the central executive network: replication from DCM. Neuroimage 2014;99:180-90.

39. Westerhausen R, Moosmann M, Alho K, et al. Identification of attention and cognitive control networks in a parametric auditory fMRI study. Neuropsychologia 2010;48:2075-81.

40. Koelewijn T, Bronkhorst A, Theeuwes J. Attention and the multiple stages of multisensory integration: a review of audiovisual studies. Acta Psychol (Amst) 2010;134:372-84.

41. Calvert GA, Thesen T. Multisensory integration: methodological approaches and emerging principles in the human brain. J Physiol Paris 2004;98:191-205.

42. Wallace MT, Meredith MA, Stein BE. Integration of multiple sensory modalities in cat cortex. Exp Brain Res 1992;91:484-8.

43. Micoulaud-Franchi JA, Vaillant F, Lopez R, et al. Sensory gating in adult with attention-deficit/hyperactivity disorder: event-evoked potential and perceptual experience reports comparisons with schizophrenia. Biol Psychol 2015;107:16-23.

44. Fostick L. The effect of attention-deficit/hyperactivity disorder and methylphenidate treatment on the adult auditory temporal order judgment threshold. J Speech Lang Hear Res 2017;60:2124-8.

45. Romei V, Murray MM, Merabet LB, et al. Occipital transcranial magnetic stimulation has opposing effects on visual and auditory stimulus detection: implications for multisensory interactions. J Neurosci 2007;27:11465-72.

46. Santangelo V, Spence C. Multisensory cues capture spatial attention regardless of perceptual load. J Exp Psychol Hum Percept Perform 2007; 33:1311-21.

47. Keil J, Senkowski D. Neural oscillations orchestrate multisensory processing. Neuroscientist 2018;24:609-26.

48. Keil J, Müller N, Ihssen N, et al. On the variability of the McGurk effect: audiovisual integration depends on prestimulus brain states. Cereb Cortex 2012;22: 221-31.

49. Hipp JF, Engel AK, Siegel M. Oscillatory synchronization in largescale cortical networks predicts perception. Neuron 2011;69:387-96.

50. Gonen-Yaacovi G, Arazi A, Shahar N, et al. Increased ongoing neural variability in ADHD. Cortex 2016;81:50-63.
51. Raichle ME, Snyder AZ. A default mode of brain function: a brief history of an evolving idea. Neuroimage 2007;37:1083-90; discussion 1097-9.

52. Sutcubasi B, Metin B, Kurban MK, et al. Resting-state network dysconnectivity in ADHD: a system-neuroscience-based meta-analysis. World J Biol Psychiatry 2020;21:662-72.

53. Chen T, Michels L, Supekar K, et al. Role of the anterior insular cortex in integrative causal signaling during multisensory auditoryvisual attention. Eur J Neurosci 2015;41:264-74.

54. Hyde KL, Peretz I, Zatorre RJ. Evidence for the role of the right auditory cortex in fine pitch resolution. Neuropsychologia 2008, 46:632-9.

55. Lehmann C, Herdener M, Esposito F, et al. Differential patterns of multisensory interactions in core and belt areas of human auditory cortex. Neuroimage 2006;31:294-300.

56. Beauchamp MS. See me, hear me, touch me: multisensory integration in lateral occipital-temporal cortex. Curr Opin Neurobiol 2005; 15:145-53.

57. Cortese S, Castellanos FX, Eickhoff CR, et al. Functional decoding and meta-analytic connectivity modeling in adult attention-deficit/ hyperactivity disorder. Biol Psychiatry 2016;80:896-904

58. Gargaro BA, Rinehart NJ, Bradshaw JL, et al. Autism and ADHD: how far have we come in the comorbidity debate? Neurosci Biobehav Rev 2011;35:1081-8.

59. van Laarhoven T, Stekelenburg JJ, Vroomen J. Increased sub-clinical levels of autistic traits are associated with reduced multisensory integration of audiovisual speech. Sci Rep 2019;9:9535.

60. Stevenson RA, Siemann JK, Woynaroski TG, et al. Evidence for diminished multisensory integration in autism spectrum disorders. J Autism Dev Disord 2014;44:3161-7.

61. Keane BP, Rosenthal O, Chun NH, et al. Audiovisual integration in high functioning adults with autism. Res Autism Spectr Disord 2010; 4:276-89.

62. Van Der Smagt MJ, Van Engeland H, Kemner C. Brief report: can you see what is not there? Low-level auditory-visual integration in autism spectrum disorder. J Autism Dev Disord 2007;37:2014-9.

63. Martínez K, Martínez-García M, Marcos-Vidal L, et al. Sensory-tocognitive systems integration is associated with clinical severity in autism spectrum disorder. J Am Acad Child Adolesc Psychiatry 2020, 59:422-33. 